\title{
Epidemic spastic paraparesis in Bandundu (Zaire)
}

\author{
H CARTON,* KAZADI KAYEMBE, $†$ KABEYA $\ddagger$ ODIO, $\ddagger$ A BILLIAU \\ K MAERTENS $\|$
}

From the Department of Neurology, Catholic University of Leuven, Belgium, ${ }^{*}$ Centre Neuropsychopathologique University of Kinshasa, Zaire, $\uparrow$ Hôpital de Panzi, Bandundu, Zaire, $\ddagger$ Rega Institute, Catholic University of Leuven, Belgium,§ Department of Ophthalmology, University of Kinshasa, Zaire \|

SUMMARY Epidemiological findings of twenty sporadic cases of epidemic spastic paraparesis (buka-buka) in three areas of Bandundu (Zaire) are reported. These findings suggest the involvement of an infectious agent and do not support the hypothesis of a dietary cyanide intoxication, which has been advanced to explain the outbreak of a very similar disease (Mantakassa) in Mozambique.

Acute as well as chronic spastic paraparesis of unknown origin is much more common in tropical countries than in temperate climates. Deficiencies or toxicities due to primitive diets as well as infectious agents have been implicated. ${ }^{1}$ Sometimes these diseases occur in epidemics. The earliest documented cases of epidemic spastic paraparesis in the Bandundu region of Zaire occurred in 1928 and were reported by Trolli. ${ }^{2}$ Ever since there have been new outbreaks affecting hundreds of cases in several areas of Bandundu, the latest ones in 1978 and $1981{ }^{3}$ Between epidemics, there have been new sporadic cases every year. The disease is characterised by a sudden onset of bilateral spastic paresis of the lower limbs, often associated with speech disorders and visual complaints. Recovery may be complete but some patients are left with a typical spastic gait, and more rarely with dysarthria. The aetiology remains unknown. However, the clinical features and the disease course are very similar to those of an illness occurring in Mozambique, named Mantakassa. ${ }^{4}$ An outbreak in 1981 of more than 1,000 cases was suspected to be the consequence of chronic cyanide intoxication due to consumption of insufficiently detoxificated cassava. ${ }^{5}$ No viruses were found. The epidemiological findings in Bandundu described here fail to support the intoxication hypothesis but rather suggest participation of an infectious agent.

\section{Material and methods}

Bandundu is one of the eight administrative regions of the republic of Zaire (fig 1). It is situated east of Kinshasa, between $2^{\circ}$ and $8^{\circ}$ south and $16^{\circ}$ and $20^{\circ}$ east. The region is

Address for reprint requests: $H$ Carton, Dept of Neurology, Akademisch Ziekenhuis Gasthuisberg, B $\mathbf{3 0 0 0}$ Leuven, Belgium.

Received 30 August 1985.

Accepted 28 September 1985 covered essentially by a savanna highland of $900 \mathrm{~m}$ altitude, cut by forest galleries along the multiple rivers running in a south-north direction.

Twenty patients residing in three localities more than $200 \mathrm{~km}$ apart, and belonging to three different tribes in Southern Bandundu were examined by two neurologists and one ophthalmologist. The histories were taken either in one $c$ of the major languages of Zaire (Lingala) or in the locap os languages: Kiyaka with the aid of a Muyaka physiciaro Tshitshokwe with the aid of the local schoolhead and $K$ 昌 suku with the aid of a local health worker. These patien were not the victims of a major outbreak but rather sporadie $\rightarrow$ cases. In the village of Kidima, the local health worker indis cated all cases suspected of having or having presented with

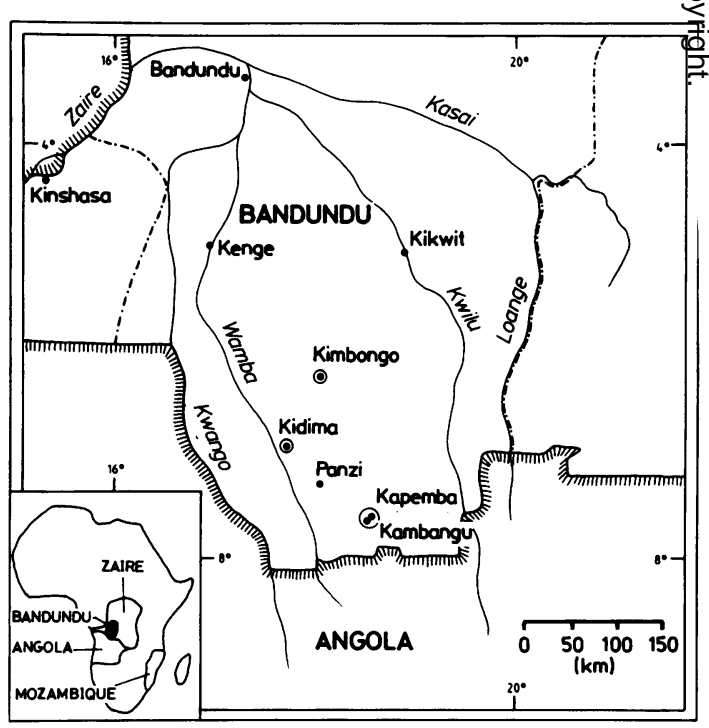

Fig 1 Map of the Bandundu Region in Zaire. The neuroepidemiological study was conducted in Kidima, Kapemba and Kambangu and in Kimbongo (circles). 
acute spastic paraparesis. All these patients were screened by the physician in charge of the primary care in that administrative zone (Dr Kabeya). Typical as well as doubtful cases were finally examined in the village by the neurologists and the ophthalmologist, and the diagnosis was either confirmed or discarded. The criteria for a case to be included were a typical history with sudden onset, complete or incomplete recovery, and the presence of pyramidal signs in the lower limbs. In Kapemba, a small village of fifteen families, in addition to history taking and examination of the patients, medical information was obtained and the ties of kinship established of every inhabitant of the village. The huts occupied by the patients at the onset of the disease could also be localised. Several patients coming from the surroundings of Kapemba were examined at the infirmary of Kambangu. Finally in the region of Kimbongo, where an important epidemic took place in 1978 , a few recent cases were examined in the local infirmary.

The neurological examination was as complete as possible, but hearing was examined with a watch and vibration sense was not tested. A general inspection for dermatological abnormalities and a brief physical examination including lung and heart auscultation and blood pressure examination were also performed. The ophthalmological examination was performed as follows: Visual acuity: outside, with a decimal Snellen chart at $5 \mathrm{~m}$; Colour vision: outside, with the Ishihara test, plates 18 to 24 for illiterates; Direct and indirect pupillary reflexes: in a dark room (inside a hut) with a torch lamp; Eye position: with a torch lamp, by studying the corneal light reflex at primary position and after occlusion; Eye movements: with the torch lamp, at six positions; Convergence: by approaching the torch lamp; Anterior segment: with a portable Kowa slitlamp, magnification $15 \times$, working from a car battery; Funduscopy: with a Welch-Allyn direct ophthalmoscope before and after pupil dilatation with Mydriacyl (R); Special attention to the colour of the optic disc, the visibility of the lamina cribrosa, the margins of the optic disc, the striation of the retina near the optic disc.

Blood was drawn from 18 patients and three controls, cerebrospinal fluid (CSF) was obtained from 10 and stool from two patients. In the CSF albumin and gammaglobulins were determined by nephelometry. Protein gel electrophoresis and isoelectrofocusing of the CSF were performed according to the method described by Delmotte. ${ }^{6}$ Blood and stool samples are being stored for possible future analysis. So far only tests for the possible involvement of the virus associated with AIDS (HTLV-III/LAV) have been done. Serum samples were tested for possible-associated reverse transcriptase as described by Billiau et $a l^{7}$ The supernatant fluids were tested for antibody by radio-immunoprecipitation, immunofluorescence (test done on isolated Ig fractions, by courtesy of Prof. L. Montagnier, Institut Pasteur, Paris) and ELISA (test done on total serum or on isolated Ig fractions, by courtesy of Dr M De Ley, Rega Institute, Leuven).

\section{A Case descriptions}

\section{PATIENTS AT KIDIMA}

Kidima is a large village in the Southern Bandundu region. It has 804 Bayaka inhabitants living in about 180 huts. The village is situated on the crests of a group of hills at about
$900 \mathrm{~m}$ height. Drinking water is supplied by several wells situated not more than $600 \mathrm{~m}$ outside the village. The women's work consists of carrying water, cultivating the fields, preparing cassava (manioc) and cooking. The men's task is mainly hunting and burning wooded areas to prepare fields for crop raising. Famine has not been present during recent years. Food is abundantly available, mainly consisting of cassava, corn, and vegetables. The villagers own numerous chickens and goats, which move freely from one area to another. Chicken meat is consumed even if obtained from birds that died from fowl plague. All members of a family eat their food out of a common jar, but the husband is first served and left-overs of the day before are eaten by the wife and children. Children always accompany their mother in her different occupations. If a man has only one wife, they dwell and sleep in the same hut. However, polygamy is still prevalent, each wife then occupying with her children her own hut. The Bayaka adhere to the matriarchal system. Epidemic spastic paraparesis has not been noticed in this village for at least 25 years until the arrival at the village of patient MM in 1977.

1 Patient MM is a female born in 1954 in Kidima. In 1972 she moved with her husband to the neighbouring village of Kasongo Bolumbu, where she fell sick in May 1977. Subsequently she was brought back to Kidima, to be taken care of by her parents, with whom she has been living ever since. Her disease started with a coldish sensation in the lower legs, followed by pain in the feet and knees which lasted one week. This episode was followed by shaking and acute paralysis of the lower limbs (clonus?). During the days or weeks preceding the illness no malaise, fever, headache or diarrhoea had been noticed. The parents as well as the two children of MM, one born before and one after her return to Kidima, are all in good health.

Clinical examination: Cranial nerves were normal. Gait was spastic. Muscle strength was slightly diminished in the $\mathrm{mm}$. triceps, extensor carpi and abductor digiti of both upper limbs, with hyperreflexia and positive Hoffmann-Trömner reflex. Examination of the lower limbs revealed moderate paresis of the flexors of the hip, the hamstrings and the dorsal flexors of the foot. Patellar reflexes were excessively brisk, with ankle clonus. The abdominal reflexes were present and the plantar reflexes equivocal. No atrophy or fasciculations were observed. Touch, pin-prick and position senses as well as coordination were unaffected. Eye examination: Vision: 1 O.U.; Ishihara: normal; Fundus: the upper, lower and nasal margins of the optic disc were blurred.

2 Patient $M A$ is a female born in 1954 in Kidima and the third living wife of the father of patient MM. Her disease started one month after the arrival of $\mathrm{MM}$ who came to live on the same parcel (but not in the same house) as MA. Hence she is the first non-imported case in the village. Shortly after she developed the first symptoms, a fowl plague episode occurred among the chickens in the village. She has two children, 7 and 13 years old, both in good health.

Clinical examination: Cranial nerves: hearing was diminished on the right side. The patient had a scissoring gait, and walking was only possible with assistance. Strength in the upper limbs was normal but reflexes were brisk. Strength in the lower limbs was reduced and patellar reflexes excessively brisk: Achilles reflex elicited clonus. Abdominal reflexes were present but a bilateral Babinski sign was noted. Touch, pin-prick and position senses as well as coordination were 
unaffected. Eye examination: Vision: 1 O.U.; Ishihara: normal; Fundus: normal.

3 Patient MaKe is a female born in 1952 in Kidima, mother of patients no 4 and no 5 and of two other children in good health. An episode of acute spastic paralysis occurred in September 1982, three months after her return to Kidima after a stay of 10 years in Kinshasa. The onset of the disease was characterised by "twitches" in the lower limbs and paraparesis. No malaise, fever, diarrhoea or visual complaints at the onset or during the preceding days or weeks are recollected. The patient completely recovered within 3 weeks.

Clinical examination: Patellar reflexes were brisk bilaterally. Achilles reflexes elicited bilateral clonus. Bilateral Babinski sign was present. Eye examination: Vision: 1 O.U.; Ishihara: normal; Fundus: normal

4 Patient MaKu is a boy born in 1976 in Kinshasa, son of MaKe (patient no 3), living with his mother in Kidima. An acute episode of spastic paralysis occurred in November 1983. The patient slowly recovered and eventually was able to walk without assistance. However, in June 1984, a relapse occurred, this time with dysarthria and more pronounced paresis of the lower limbs. There has been limited recovery since.

Clinical examination: The patient was unable to walk independently and when assisted he exhibited a very spastic scissoring gait. Pronounced dysarthria was present. Muscle strength of the upper limbs was normal but pathologically brisk tendon reflexes were found. Muscle strength of the lower limbs was reduced in hip flexors, hamstrings and dorsal flexors of the feet. Patellar reflexes were brisk. A sustained ankle clonus and a bilateral Babinski sign were found. Eye examination: Vision: 1 O.U.; Ishihara: normal; Fundus: normal.

5 Patient $P K$ is a girl born in Kinshasa in 1980, sister of patient no 4. Spastic paralysis occurred in June 1984, simultaneously with the relapse in her brother, patient no 4 . The onset was characterised by shaking and abrupt paralysis of the lower legs, with pain on palpation of the muscles. Gait recovered incompletely within one week. No prodromal malaise, fever, cough or intestinal problems were recollected by the mother.

Clinical examination: Muscle strength and reflexes in the upper limbs were normal; muscle strength in the lower limbs was reduced. Patellar reflexes were bilaterally symmetrically hyperreflexic. Achilles reflexes elicited bilateral clonus. Plantar reflexes were equivocal. Abdominal reflexes were absent. Eye examination: Vision: 1 O.U.; Ishihara: normal; Fundus: normal.

6 Patient $M u K u$ is a female born in 1960 in Kidima, mother of a child of 23 months in good health. She is the neighbour of patients no 3 , no 4 and no 5 . The spastic paresis started in July 1984, shortly after onset of the disease in patient no 5. Pain around the knees during one week was followed by trembling and paresis of the lower legs, which became complete in about one day. At that time she also had blurred vision and saw spark-like spots moving before her eyes. These symptoms subsided as soon as she was again able to walk. Recovery took about two months but remained incomplete. No preceding illness is recollected.

Clinical examination: Muscle strength and reflexes in the upper limbs were normal, but the patient has a scissor gait with a slight pyramidal paresis in the lower limbs, absent abdominal reflexes, hypertonia and hyperreflexia, and equivocal plantar reflexes. Eye examination: normal except for white light reflexes along the peripheral veins.

7 Patient $B V$ is a boy born in Kidima on 25 December 1977, son of MuKi (patient no 8). In August 1983, following two days of shaking of the lower legs, he became completely paraplegic within 48 hours. Urinary incontinence and hypophonia were present. During the following month, his voice returned and walking again became possible. However he relapsed in July 1984, at about the same time that his mother became paraplegic.

Clinical examination: Moderate dysarthria; normal muscle strength in the upper limbs with hyperreflexia. Walking was impossible as a consequence of a severe spastic paraparesis with very brisk patellar reflexes, sustained ankle clonus and bilateral Babinski sign. The abdominal reflexes were present. Eye examination: Vision: 1 O.U.; Ishihara: normal: Fundus: normal.

8 Patient $M u K i$ is a female, 33 years old, mother of BV (patient no 7). The illness started in July 1984, with pain in the knees, followed by shaking of the legs for two days and severe paraparesis on the third day, accompanied by dysarthria. At the time her legs started to shake, she observed light sparks moving before the eyes and had blurred vision first of the right eye, the next day also of the left eye. She also complained of horizontal diplopia. Smell was lost for one week.

Clinical examination: The patient had a slight dysarthria 0 के and a spastic gait but was able to walk without assistance. Muscle strength and reflexes were normal in the upper limbs. A moderate spastic paresis was present with patellar hyperreflexia and bilateral ankle clonus. The abdominal reflexes were absent; the plantar reflexes were equivocal. Eye examination: Vision: 1 O.U.; Ishihara: normal: Fundus: normal. Eye movements: normal.

A schematic groundplan of Kidima with location of the dwellings of every patient as well as the year of onset of the spastic paraparesis is given in fig 2 .

\section{PATIENTSAT KA PEMBA}

Kapemba is a small Batshokwe village of 45 inhabitants living in 15 huts, situated near a small forest at about $1,000 \mathrm{~m}$ height. Water is taken from the nearby Tshikweso river. About two months before our arrival, the people had moved and rebuilt the entire village at a distance of about $500 \mathrm{~m}$ from its original location. Chickens and goats wandered around freely. Only a minority of a little more than 100,000

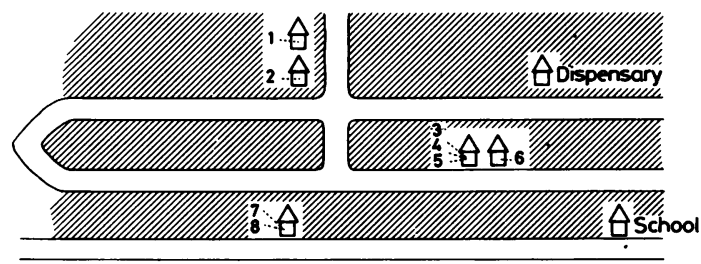

$100 \mathrm{~m}$

Fig 2 Simplified map of Kidima, a village of about 180 huts. Patient no 1 and 2 became paretic in 1977, patients no 3 in 1982, no 4 fell sick in 1983 and again in 1984, no 5 and no 6 in 1984. Patient no 7 became paretic in 1983 and relapsed in 1984, no 8 fell sick also in 1984. 
Batshokwe live in the deep south of Bandundu (Zaire), the majority occupy a territory in neighbouring Angola. However, family ties between the two groups remain strong and they still intermarry. Epidemic spastic paraparesis does not spare their Angolan brothers. Batshokwe life-style, working and feeding habits have much in common with those of the Bayaka. They also adhere to the matriarchal system.

9 Patient $K W$ is a 22-year-old female born in Kapemba, who at the age of 7 years developed acute spastic paresis. Considerable improvement occurred over a period of several months and finally she was able to walk to the river and to work on the fields. In $1983 \mathrm{KW}$ was still living in her father's hut when her aunt with her four children moved into the same house. Two of these children were to fall sick a few months later. During the summer of 1984, still living in the same hut as her two spastic nephews, $\mathrm{KW}$ had a relapse: while walking to the river, she felt a painful sensation in the legs. Trying to return to the village, she stumbled several times, probably because her legs were shaking. She felt feverish but had no headache, malaise or other systemic complaints. For several days she was incontinent of urine. Over a period of two weeks, she noticed sparks moving in front of her right eye. She also had double vision for 4 to 5 days. This time, recovery was less complete.

Clinical examination: Gait was very spastic, and almost impossible without assistance. Muscle strength and reflexes in the upper limbs were normal. Muscle strength in the lower limbs was reduced in the hip flexors and hamstrings. Patellar reflexes were brisk. Achilles reflexes elicited sustained clonus. A bilateral Babinski sign was present. Touch, pinprick and position senses were normal. Coordination was normal. Eye examination: Fundus: small excavation of the optic disc, where the lamina cribrosa could be seen; Iris: depigmentation of the lower part. Vision: 1 O.U.; Ishihara: normal. Eye movements: normal.

10 Patient MaKa is a 12-year-old boy born in Kapemba. $\mathrm{He}$ is the oldest of five children (two boys and three girls). One girl died of an unknown disease. When the father died of an unspecified disease in 1983, the mother and her four children, all in good health at that time, moved into the hut of their uncle, the father of KW (patient no 9). Two to three months after their move (still in 1983), MaKa and his brother developed acute spastic paraparesis. While coming back from the river, MaKa felt electrical sensations around his knees and immediately started to tremble in his lower legs. He fell several times while trying to reach the village. The paraplegia was complete in less than one week. He was also dysarthric for several days. There were no micturition problems, no eye, smell or hearing problems in the acute phase. Before and during the acute phase, no systemic complaints of fever or malaise were noted. After several months, there was incomplete recovery.

Clinical examination: Cranial nerves were normal, except for a slight nasal voice. The patient walked slowly without aid, with a spastic scissoring gait. Muscle strength, tone and reflexes of the upper limbs were normal. A moderate spastic paresis of the lower limbs with ankle clonus and bilateral Babinski sign were found. Touch, pin-prick and position senses as well as coordination were normal. Eye examination: Vision: 1 O.U.: Ishihara: normal; Fundus: normal. $\mathrm{KW}, \mathrm{MaKa}$ and his brother are the only three cases to have occurred in Kapemba. All three contracted the disease in the same hut. Before moving into that hut, MaKa and his brother lived in a hut at the opposite side of the village about $100 \mathrm{~m}$ away.

\section{PATIENTS EXAMINED IN KAMBANGU AND IN KIMBONGO}

Seven patients from neighbouring villages were examined in the Dispensary of Kambangu (near Kapemba). Three patients belonging to the Basuku tribe were examined at the Hôpital-Dispensaire of Kimbongo $200 \mathrm{~km}$ to the north. Their histories and clinical symptoms and signs will not be reported in extenso; the data will be used to draw a general picture of epidemic spastic paraparesis in Bandundu.

Patients no $11(\mathrm{ME})$ and no $12(\mathrm{Ki})$ are cousins and more than 50 years old. They both developed the disease more than $\mathbf{4 0}$ years ago. Patient no 13 (MwK) is $\mathbf{4 5}$ years old and became acutely paralysed at the age of 6 . None of these three patients ever had a relapse, or have noticed any progression of the spastic paraparesis. Patient no 14 (Kal) is a 28-year-old half-sister of ME who became suddenly paralysed one week after the delivery of a normal baby.

Patient no 15 (Kat) is a 20-year-old female who, about a year ago, stayed for several months in the village of her father living in a house together with several children presenting signs of incompletely recovered spastic paraparesis. One week after her returning to the village of her husband, she developed acute spastic paraparesis. At that time, there were no other cases in her village. Patient no 16 (Wan) is a boy born in 1979, developing the disease in 1984. Patient no 17 (NgMa) is a 6-year-old girl who developed the disease one day after the onset of diarrhoea and fever. She had transitory hearing loss. Patient no $18(\mathrm{LoBw})$ and patient no 19 (MaMu), mother and son, developed the disease on the same day of July 1984 . Four other children as well as the father remained unaffected. Patient no 20 (NuTi) developed spastic paralysis at the age of 23 . He is the sole male encountered to have developed acute spastic paraparesis in adulthood.

Patients no 18, no 19 and no 20 belong to the Basuku tribe. In 1978, an epidemic was rampant in this population living around Kimbongo. According to the hospital files between 12 July 78 and 24 August 78, 67 patients were taken care of at the Hôpital/Dispensaire/Maternité of Kimbongo with typical acute spastic paraparesis. Nineteen were adult females, 27 were young girls and 21 were young boys under the age of 11 except for one boy of 16 . Temperature was taken in all patients in the acute phase but fever was never detected. No diarrhoea was noted and all had good appetite. About half the patients could be dismissed after a few weeks without apparent disability. However, one patient died after a week-long progressive ascending paralysis, involving legs, arms and respiratory muscles and finally the muscles of deglutition. She remained alert till she died of respiratory failure.

\section{B General clinical picture of epidemic spastic paraparesis in Bandundu}

Nine out of 20 patients examined were adults, most in their twenties: one male aged 22 and 8 females between 19 and 39 . All females were mothers of at least one child, but none was pregnant at the time of disease onset. Eleven patients were children: seven boys aged 4 to 11 , and four girls aged 4 to 7 
(fig 3). About half of these children had been vaccinated against measles and poliomyelitis, which probably corresponds to the proportion of vaccinees in the whole child population. Reliable disease histories were obtained from 15 patients. With only one exception, none of them could recall a preceding systemic illness with any headache, malaise, fever, loss of appetite, abdominal complaints or other flu-like symptoms. One patient had diarrhoea on the day before onset. All patients, except one, mentioned painful dysaesthesia in the lower limbs, involving either the whole leg or being limited to the soles of the feet or around the knees in particular. This pain preceded onset of paralysis by a time interval of a few hours to 7 days. The onset of paralysis was abrupt in all cases. It was preceded or accompanied by shaking or tremor (clonus ?) of the lower limbs. Several patients mentioned suddenly falling on their way back from the river. Trembling of the arms was also mentioned in $5 / 15$ patients. Weakness of the arms was only recalled by $3 / 15$ patients. It was rather slight and short-lasting, while paralysis of the lower limbs was said to have been complete in more than half the patients. Maximal weakness was attained in a few hours to about one week. One of the 15 patients mentioned loss of smell for about one week at the onset of disease, two mentioned transient deafness, four loss of bladder control for about a week and seven dysarthria (slurred speech to almost complete aphonia). Eight, finally, recalled visual disturbances, most often blurred vision for weeks or months. Some patients reported sensations of moving sparklike light spots lasting for several days. Transient diplopia was reported by three patients. Some degree of recovery always occurred. It started a few days to several weeks after onset and was near-complete in many cases. We obtained only one history of a patient, a child, having died in the acute phase of the disease completely paralysed. Disease episodes indicative of relapse occurred in three patients. Two of these were children in whom apparent relapse occurred one year after the first episode. In a 22-year-old female, it occurred 15 years after the first episode. These relapses consisted of a sudden exacerbation of the residual signs, but in one patient there was marked dysarthria, which had never been a complaint before and which remained from the second episode onward. In all other patients, including three with a history of at least $\mathbf{4 0}$ years, the clinical picture has remained unchanged after the period of partial recovery.

Twenty patients underwent neurological and ophthalmological examinations. The most pertinent neurological

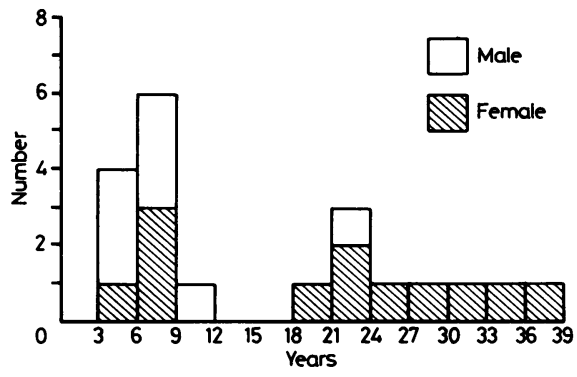

Fig 3 Distribution of age at onset of 20 sporadic cases of epidemic spastic paraparesis of Bandundu. findings are given in the table. Persisting ocular findings were scarce and limited to retinal vessel vasculitis and one case of protanopsia in a female which could be interpreted as acquired dyschromatopsia due to a lesion of the optic nerve. Retinal fibre defects as observed in demyelinating optic neuropathy were not observed. General physical examination failed to reveal signs of malnutrition, skin disease, lymphoadenopathy, pulmonary or cardiac anomalies.

\section{Laboratory findings}

Laboratory investigations on serum samples were restricted to screening for HTLV-III/LAV or antibodies against this virus. None of the blood samples showed evidence of or presence of a circulating retrovirus (assay for particleassociated reverse transcriptase). One out of 10 serum samples contained a low titre of anti-HLV as detected by ELISA. Tests done on isolated Ig fractions were all negative. No abnormal immunoglobulin fractions were detected in the cerebrospinal fluid by gel electrophoresis or electrofocusing.

\section{Discussion}

Acute spastic paraparesis, also named Buka-Buka, Kitondji or Konzo in the local languages, was first described in 1938 by Trolli. $^{2}$ It occurs in local outbreaks between May and December in several areas in Bandundu and neighbouring Angola. The earliest epidemics documented ${ }^{89}$ occurred in 1928, 1932 and $\overparen{\triangle}$ 1937. One of the latest outbreaks was observed in 1981 in Masi-Manimba affecting about 200 women and children. ${ }^{3}$ However, sporadic cases and small clusters occur between epidemics. For example, since a first upsurge in the area of Kimbongo in 1978 leading to the hospitalisation of 67 patients, five too seven new cases are seen each year at the? Hôpital/Dispensaire. In Zaire, the disease appears to be restricted to the administrative region of Bandundu but it is also rampant in the neighbouring northern part of Angola.

The clinical picture is conspicuously uniform. Without preceding illness or prodromal symptoms, the patients start complaining of painful paraesthesiae in the legs, and within a few hours or days develop weakness of the lower limbs, often with visual

Neurological findings in 20 patients with spastic paraparesis of bandundu

\begin{tabular}{lc}
\hline Spastic gait & 18 \\
Gait not possible without help & 2 \\
Arms: weakness & 0 \\
$\quad$ hyperreflexia & 7 \\
Legs: weakness & 13 \\
$\quad$ hyperreflexia & 20 \\
$\quad$ Babinski sign & 12 \\
Absent abdominal reflexes & 4 \\
Unilateral deafness & 1 \\
Dyarthria & 4 \\
Incontinence for urine & 1 \\
Mental/cerebellar/sensory abnormalities & 0 \\
\hline
\end{tabular}


complaints and dysarthria, rarely with hearing loss and incontinence of urine. Fever, malaise or other systemic complaints are not part of the clinical syndrome. After a few days or weeks, the patients start to recover muscle strength but many of them are left with a spastic gait. Dysarthria is permanent in only a few patients and permanent ocular lesions are very rare. No cerebellar, sensory or peripheral nerve signs are evident at least during or after the recovery phase. The clinical picture always is and remains strikingly symmetrical. A monophasic course appears to be the rule, although in a few cases an apparent relapse of the disease was observed. Epidemic as well as sporadic cases mostly appear between May and October which corresponds to the dry season in that region of Africa. The age of incidence shows a bimodal distribution: boys and girls between 4 and 12 years of age and women of reproductive age are preferentially affected (fig 2). On the other hand, adult males only rarely contract the disease. Very young children and elderly persons of both sexes appear to be spared.

Clusters of spastic cord disorders, often occurring in epidemics, have been described in many parts of the tropical world. ${ }^{2}$ Mantakassa or Spastic Paraparesis of Mozambique ${ }^{4}$ appears to be endemic in some parts of that country and it assumed epidemic proportions in 1981 when more than 1,000 cases occurred. It is clinically similar if not identical to Spastic Paraparesis of Bandundu. It shows the same mode of onset, the same clinical features and disease course, the same vulnerability of children of both sexes and of young women, and the same relation to the dry season as Spastic Paraparesis of Bandundu. A chronic cyanide intoxication due to consumption of inadequately detoxificated cassava has been implicated. ${ }^{5}$ Cassava constitutes about $80 \%$ of the staple food in the affected area. In periods of severe drought, it is the only food crop to survive. When for reasons of food shortage cassava is not submitted to the lengthy process of drying in the sun, the cyanogenic glycosides are not adequately removed and the inhabitants are exposed to the risk of chronic cyanide intoxication.

Another tropical neurological disease named "Ataxic Neuropathy of Nigeria", has also been linked to chronic cyanide intoxication. ${ }^{10}$ However several features such as the slow onset and the older age of the patients as well as the commonest signs such as ataxia, muscle wasting and diminished sensory perception distinguish the disease from Spastic Paraparesis of Bandundu and from Mantakassa in Mozambique.

Spastic paraparesis of Bandundu superficially resembles lathyrism, ${ }^{11}$ a disease occurring in central India, which periodically assumes epidemic proportions. Lathyrism is also characterised by spastic gait and pyramidal signs in the lower limbs with con- servation of other neurological functions. However, some important differences exist. Thus, although the onset of lathyrism is often acute, subacute and slowly progressive cases are not rare, and a recovery phase appears to be exceptional. Visual complaints and dysarthria are not part of the clinical syndrome. Furthermore, when adults are affected by lathyrism, this occurs more frequently in males than in females, and unlike Spastic Paraparesis of Bandundu, women of reproductive age appear to be resistant to the disease while postmenopausal women are seldom affected. Consumption of the peas of Lathyrus sativus, which contains B-(N) oxalylaminoalanine is said to be responsible for this syndrome.

A "spastic paraplegic syndrome" described by Mani et $a^{12}$ in South India appears to be a different entity. The age of onset of symptoms is from 9 to 65 years. Males are more affected than females and the development is insidious over weeks and months. Objective evidence of sensory deficit occurs in over $80 \%$ of the patients and peripheral neuropathy in about one fourth. The aetiology of South Indian Paraplegia remains unknown.

Jamaican Neuropathy is a chronic disorder of adults featuring pyramidal tract signs in different combinations with retrobulbar neuropathy, nerve deafness, posterior column signs and lower motor neuron lesions. ${ }^{13}$

Sporadic cases of acute spastic paraparesis or of neuromyelitis optica of unknown origin and clinically to some extent similar to the disease under study, are not uncommon in tropical Africa ${ }^{1}$ and amongst blacks in South Africa. ${ }^{14}$ A geographic isolate of Tropical Spastic Paraparesis in Tumaco, Colombia, was recently described by Roman et al. ${ }^{15}$ Again this is a different disease entity. It is a slowly progressive disorder, typically beginning in middle-aged persons of both sexes. The aetiology is unknown. However, dysarthria, the typical age distribution and preferential vulnerability of women of reproductive age so typical for acute spastic paraparesis in Bandundu and in Mozambique, are seldom or never mentioned. Likewise, speech disorders were not encountered in children with acute spastic paraparesis examined by one of the authors in Rwanda.

In conclusion, on clinical and epidemiological grounds, it appears that Acute Spastic Paraparesis of Bandundu is most probably the same disease as Mantakassa in Mozambique, but different from other epidemic spastic syndromes in the tropics, as well as from sporadic cases of acute spastic paraparesis in other parts of Africa.

The aetiology of Spastic Paraparesis of Bandundu remains obscure. Several not mutually exclusive hypotheses can be advanced: nutritional deficiencies, environmental neurotoxins and infection with a 
specific agent. They will successively be discussed in the light of some of our findings which may help to define better the aetiology. The more relevant amongst these findings are: (1) the disease is not uniformly spread throughout the villages but rather occurs in limited numbers of foci; (2) the disease has a clear age and sex preference: boys and girls between 4 and 12 years and females between 20 and 40 ; (3) if one affected case is present or introduced in a community, children or adult females living in the proximity (that is in the same or an adjacent hut) seem to run a higher risk of acquiring the disease; (4) spread to family members or neighbours is still possible after an interval of one to several years; (5) relapses are rare but do occur.

A first possible aetiology to be discussed is nutritional deficiency. Some degree of undernourishment and of unbalanced diets may always be suspected in these regions. Furthermore, cassava, which is the main food component, is a poor source of vitamin B, in particular of riboflavin. However, in our Bandundu field study, no evidence could be found for malnutrition or for temporal changes in feeding habits neither at the village level nor in families with affected members. Actually the patients and their families insisted on always having had available plenty of cassava, corn and earthnuts, and no mucocutaneous abnormalities suggestive of riboflavin deficiency were detected on physical examination.

A second aetiology to be considered is food-related toxic factors, especially chronic cyanide intoxication. Indeed, as in Mozambique, cassava constitutes the staple food in Bandundu and most cases of Acute Spastic Paraparesis did occur in the dry season. It should be mentioned however that in Bandundu the cassava is not only detoxificated by sundrying as is done in the affected region of Mozambique, but mainly by soaking in water which is a much more efficient method. Several other points argue against the implication of chronic cyanide intoxication in spastic paraparesis in Bandundu. The patients denied having had to consume inadequately prepared cassava or to limit their diet to this root exclusively. Furthermore, the cassava hypothesis would still not explain the occurrence of the disease in a few limited foci of close neighbours and the apparent transmission in some households with intervals of years. Even for Mantakassa in Mozambique, the role of cyanide intoxication has been questioned ${ }^{16}$ on the basis of the observation that thiocyanate blood levels were high in affected and unaffected alike. Furthermore, another tropical disease named Ataxic Neuropathy of Nigeria ${ }^{10}$ has also been linked to chronic cyanide intoxication. However, several features (see above) distinguish this disease from spastic Paraparesis of Bandundu and from Mantakassa in Mozambique. If cyanide is to be held responsible for both diseases, it would be difficult to explain how two so different clinical syndromes could emerge as a consequence of a single agent.

Another neurotoxic substance to be discussed is B-(N) oxalylaminoalanine which is implicated as an aetiological factor of lathyrism in Central India. ${ }^{11}$ However, Lathyrus sativus is not cultivated in Bandundu and is not part of the diet of the population.

Clustering of the disease in families also could be the result of focal rather than a general intoxication: for example the use of some toxic herb by only a few families or negligence in preparing and storing food. Actually it is the custom in these regions that leftovers of the previous day are eaten by the women and children while the adult males receive freshly prepared food. A search for toxic herbs or other toxic products was not undertaken. Nevertheless, a focal toxicity theory would still not account for the apparent transmission of the disease between neighbours (patients no 2 and no 6) nor could it explain why one patient (patient no 5) fell sick in her own village one week after having shared the hut with partially recovered patients in another village.

The occurrence of the disease in a few limited foci of family members and close neighbours as well as the apparent transmission to outsiders who for some time are brought in the vicinity of patients (patients no 2 , no 10 , no 15 ), can best be explained by implicating a specific infectious agent. The epidemiology as $\vec{c}$ described is highly suggestive of transmission by close contact. Patients appear to be able to transmit the disease not only during the acute phase but also manyto $\overrightarrow{0}$ years thereafter (patients no 3 , no 7 , no 9). This? suggests that the infectious agent persists in the patients and is shed continuously or intermittently as it occurs in infections with various viruses of the herpes group (fam. Herpetoviridae), human Tlymphotropic viruses (HTL, fam. Retroviridae) or hepatitis-B virus (fam. Hepadnaviridae). Furthermore, a bimodal distribution of the age of maximal susceptibility has been observed in several infectious diseases. The reason why adult males remain mostly spared could be some differing social behaviour which avoids contagious contact or some hormonal protection.

The distribution in families might suggest a genetic predisposition. In our field survey, we have reconstructed the genealogical tree of every single living member of Kapemba village. It soon became clear that each inhabitant was closely related to almost all others, probably a general feature in all small communities in the Bandundu region. Against this background, there is nothing exceptional about patients no 9 and no 10 being paternal cousins when compared to the familial relationship of the other 
Kapemba inhabitants. An argument against an important genetic influence is the observation that spastic paralysis does not occur amongst the large Bayaka and Basuku population living in the city of Kinshasa.

Although our data suggest a transmission of the disease by some infectious agents, some arguments against this hypothesis should not be disregarded. First of all, except for the isolation at the Tropical Institute of Antwerp in 1978 of an ECHO virus in the stools of a patient at Kimbongo, microbiological tests performed at the Center of Disease Control at Atlanta on acute blood, CSF, urine and stools of acute patients of Bandundu ${ }^{3}$ as well as of Mozambique, ${ }^{4}$ remained negative. Likewise, our search for a HTLVIII/LAV virus remained negative, and no oligoclonal immunoglobin bands were detected in the CSF. Furthermore, all signs or symptoms of any infectious disease such as malaise, fever, cough or abdominal complaints were conspicuously absent in the histories of almost all patients of this study although patients in Mozambique did mention some general symptoms at the time of onset of the disease. Other clinical features of spastic paresis, such as its striking uniformity and its total symmetry are unusual for diseases caused by viral invasion of the central nervous system. A possible explanation would be that the neurological signs and symptoms are the consequence of a delayed immunological reaction in the central nervous system to a previous infection, as is well recognised in the peripheral nervous system for the Guillain-Barré syndrome.

A first step in elucidating the problems of aetiology and physiopathology would be the microscopic study of brainstem and spinal cord. However, the rareness of fatal outcome, the remoteness of the regions involved, the local customs and the Zaire law all make the chances of ever obtaining suitable necropsy material remote.

The authors are grateful for the collaboration and help of Sister Bangola Mudiando of the mission of Kambangu and Sister M Standaert of the Hôpital Dispensaire of Kimbongo.

\section{References}

${ }^{1}$ Spillane JD, ed. Tropical Neurology. London, Oxford University Press, 1973.
${ }^{2}$ Trolli G, Résumé des observations réunies au Kwango au sujet de deux affections d'origine indéterminée. Paraplégie spastique épidémique. Fondation Reine Elisabeth, Bruxelles, 1938.

${ }^{3}$ Anonymous, Surveillance of peripheral neuropathies. Weekly Epidemiological Records. 1982;28:213-14.

${ }^{4}$ Ministry of Health, Mozambique. Mantakassa: an epidemic of spastic paraparesis associated with chronic cyanide intoxication in a cassava staple area of Mozambique. 1 Epidemiology and clinical and laboratory findings in patients. Bulletin of the World Health Organization 1984;62:474-84.

${ }^{5}$ Ministry of Health, Mozambique. Mantakassa: an epidemic of paraparesis associated with chronic cyanide intoxication in a cassava staple area of Mozambique. 2 Nutritional factors and hydrocyanic acid content of cassava products. Bulletin of the World Health Organization 1984;62:485-92.

${ }^{6}$ Delmotte P. Gel isoelectrofocusing of cerebrospinal fluid proteins: a potential diagnostic tool. Zeitschrift für Klinische Chemie und Klinische Biochemie 1971;9:334-6.

${ }^{7}$ Billiau A, Heremans H, Allen PT, Barous S, De Somer P. Interferon inhibits L-Type Virus at a Posttranscriptional prerelease step. Archives of Virology 1975;57:205-20.

${ }^{8}$ Viléu AF. Contribution à la discussion de la paraplégie spastique épidémique du Kwango. Annales de la Société Belge de Médecine Tropicale 1942;22:309-17.

${ }^{9}$ Lucasse C. Le Kitondji, une paralysie spastique. Annales de la Société Belge de Médecine Tropicale 1952;33:393-401.

${ }^{10}$ Osuntokun BO. Cassava diet, chronic cyanide intoxication and neuropathy in the Nigerian Africans. World Review of Nutritional Diets 1981;36:141-73.

${ }^{11}$ Lala Surajnandan Prasad, Sharan RK. Lathyrism. Handbook of Clinical Neurology. Vinken PJ and Bruyn GW editors. Volume 36, 505-14. North-Holland Publishing Company, Amsterdam, 1979.

${ }^{12}$ Mani KS, Mani KJ, Montgomery RD. A spastic paraplegie syndrome in South-India. $J$ Neurol Sci 1969;9:179-99.

${ }^{13}$ Montgomery RD, Cruickshank EK, Robertson WB, McMenemey WH. Clinical and pathological observations on Jamaican neuropathy. A report of 206 cases. Brain 1964;84:425-62.

${ }^{14}$ Wallace ID, Cosnett JE. Unexplained spastic paraplegia. $S$ Afr Med J 1983;63:689-91.

${ }^{15}$ Roman GC, Roman LN, Spencer PS, Schoenberg BS. Tropical Spastic Paraparesis: A Neuroepidemiological Study in Colombia. Ann Neurol 1985;17:361-5.

${ }^{16}$ Anonymous. Epidemic Spastic Paraparesis. Lancet 1984;ii:904-5. 